\title{
Oxygen pressure measurement using singlet oxygen emission
}

\author{
Gamal E. Khalil, ${ }^{\text {a) }}$ Alvin Chang, Martin Gouterman, James B. Callis, and Larry R. Dalton \\ Department of Chemistry, The University of Washington, Seattle, Washington 98195
}

\author{
Nicholas J. Turro and Steffen Jockusch \\ Department of Chemistry, Columbia University, New York, New York 10027
}

(Received 28 December 2004; accepted 8 February 2005; published online 20 April 2005)

\begin{abstract}
Pressure sensitive paint (PSP) provides a visualization of two-dimensional pressure distributions on airfoil and model automobile surfaces. One type of PSP utilizes platinum tetra(pentafluorophenyl)porphine (PtTFPP) dissolved in a fluoro-polymer film. Since the intense $650 \mathrm{~nm}$ triplet emission of PtTFPP is quenched by ground state oxygen, it is possible to measure two-dimensional oxygen concentration from the $650 \mathrm{~nm}$ emission intensity using a SternVolmer-type relationship. This article reports an alternative luminescence method to measure oxygen concentration based on the porphyrin-sensitized $1270 \mathrm{~nm}$ singlet oxygen emission, which can be imaged with an InGaAs near infrared camera. This direct measurement of oxygen emission complements and further validates the oxygen measurement based on PtTFPP phosphorescence quenching. Initial success at obtaining a negative correlation between the $650 \mathrm{~nm}$ PtTFPP emission and the $1270 \mathrm{~nm} \mathrm{O}$ emission in solution led us to additional two-dimensional film studies using surfaces coated with PtTFPP, MgTFPP, and $\mathrm{H}_{2}$ TFPP in polymers in a pressure and temperature controlled chamber. (C) 2005 American Institute of Physics. [DOI: 10.1063/1.1896623]
\end{abstract}

\section{INTRODUCTION}

The phosphorescence originating from the ${ }^{3} T_{1}$ excited state of platinum (II) porphyrin [PtP] is well known to have a short lifetime $(\sim 100 \mu \mathrm{s})$ and a high quantum yield $(\sim 90 \%) .{ }^{1-6}$ The high phosphorescent yield results from the near unity triplet quantum yield due to the mixing between the platinum filled $d_{\pi}$ orbital and the porphyrin empty $e_{g}\left(\pi^{*}\right)$ molecular orbital, which brings strong spin coupling into the ${ }^{3} T_{1}\left(\pi, \pi^{*}\right)$ transition. ${ }^{1,2,6}$ An alternative to phosphorescence for deactivation of $\operatorname{PtP}\left[{ }^{3} T_{1}\right]$ is quenching by energy transfer of $\operatorname{PtP}\left[{ }^{3} T_{1}\right]$ to $\mathrm{O}_{2}\left[{ }^{3} \Sigma_{g}^{-}\right]$resulting in $\mathrm{O}_{2}\left[{ }^{1} \Delta_{g}\right]$, which has an emission at $1270 \mathrm{~nm}$. The quenching of PtP emission by oxygen gives rise to changes in PtP phosphorescence intensity and lifetime, and this response is the operating mechanism of pressure sensitive paint (PSP), a pressure measurement method that provides real time continuous twodimensional surface pressure data with high spatial resolution. ${ }^{4,7-9}$

An energy diagram corresponding to the interaction of PtP and oxygen under optical excitation is presented in Fig. 1. The following are the processes of interest for PSP:

$$
\operatorname{PtP}\left[{ }^{1} S_{0}\right]+h v_{\text {exc }} \rightarrow \operatorname{PtP}\left[{ }^{1} S_{n}\right]: \text { Photoexcitation to a }
$$
higher excited singlet;

(ii) $\operatorname{PtP}\left[{ }^{1} S_{n}\right] \rightarrow \operatorname{PtP}\left[{ }^{3} T_{1}\right]+$ heat: Intersystem crossing to the lowest triplet;

(iii) $\operatorname{PtP}\left[{ }^{3} T_{1}\right] \rightarrow \operatorname{PtP}\left[{ }^{1} S_{0}\right]+h v[650 \mathrm{~nm}]:$ Triplet phosphorescence;

(iv) $\operatorname{PtP}\left[{ }^{3} T_{1}\right] \rightarrow \operatorname{PtP}\left[{ }^{1} S_{0}\right]+$ heat: Intersystem crossing to ground state; and

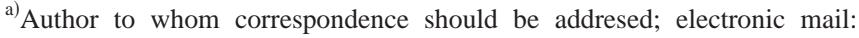
gkhalil@u.washington.edu
}

$\mathrm{PtP}\left[{ }^{3} T_{1}\right]+\mathrm{O}_{2}\left[{ }^{3} \Sigma_{g}^{-}\right] \rightarrow \mathrm{O}_{2}\left[{ }^{1} \Delta_{g}\right]+\operatorname{PtP}\left[{ }^{1} S_{0}\right]: \quad$ Triplet quenching with formation of singlet oxygen.

Process (ii) is so fast that the quantum yield of $\operatorname{PtP}\left[{ }^{3} T_{1}\right]$ is near unity. Processes (iii) and (iv) are intrinsic properties of PtP, where (iii) is much faster than (iv) as seen by a phosphorescence efficiency of $\sim 90 \%$ when no alternative triplet PtP relaxation mechanisms such as (v) are available. Competition between (iii) and (v) provides the mechanism for oxygen sensing. As oxygen concentration increases, more $\operatorname{PtP}\left[{ }^{3} T_{1}\right]$ relaxes via (v) vs (iii), hence phosphorescence is quenched. Pressure sensitive paint (PSP) utilizes this indirect measurement of oxygen concentration by examining phosphorescence of PtP and agents other than $\mathrm{O}_{2}\left[{ }^{3} \Sigma_{g}^{-}\right]$. In this article, we propose to measure oxygen concentration directly by looking at oxygen emission and use the comparison between the indirect and direct methods to validate and improve PSP performance.

The ground state of molecular oxygen is a triplet, ${ }^{3} \Sigma_{g}^{-}$. Its two lowest excited states are ${ }^{1} \Delta_{g}$ and ${ }^{1} \Sigma_{g}^{+}$, known as singlet molecular oxygen, which lie $7882 \mathrm{~cm}^{-1}$ $(\sim 1270 \mathrm{~nm})$ and $13121 \mathrm{~cm}^{-1}(\sim 760 \mathrm{~nm})$ above the ground state, respectively. In free molecular oxygen, the absorptions, ${ }^{3} \Sigma_{g}^{-} \rightarrow{ }^{1} \Delta_{g}$ and ${ }^{3} \Sigma_{g}^{-} \rightarrow{ }^{1} \Sigma_{g}^{+}$, are very weak since these transitions are both electric dipole and spin forbidden. Thus singlet oxygen production by absorption of radiation is a very inefficient process. However, the process of oxygen quenching excited photosensitizers such as $\operatorname{PtP}\left[{ }^{3} T_{1}\right]$ produces singlet oxygen very effectively via bimolecular energy transfer. The radiative lifetimes of unperturbed singlet oxygen are $45 \mathrm{~min}$ for ${ }^{1} \Delta_{g}$ and $11 \mathrm{~s}$ for ${ }^{1} \Sigma_{g}^{+}$. The observed lifetimes for ${ }^{1} \Delta_{g}$ range from milliseconds in gaseous phase to microseconds in aqueous media, whereas the lifetime of ${ }^{1} \Sigma_{g}^{+}$is much shorter 


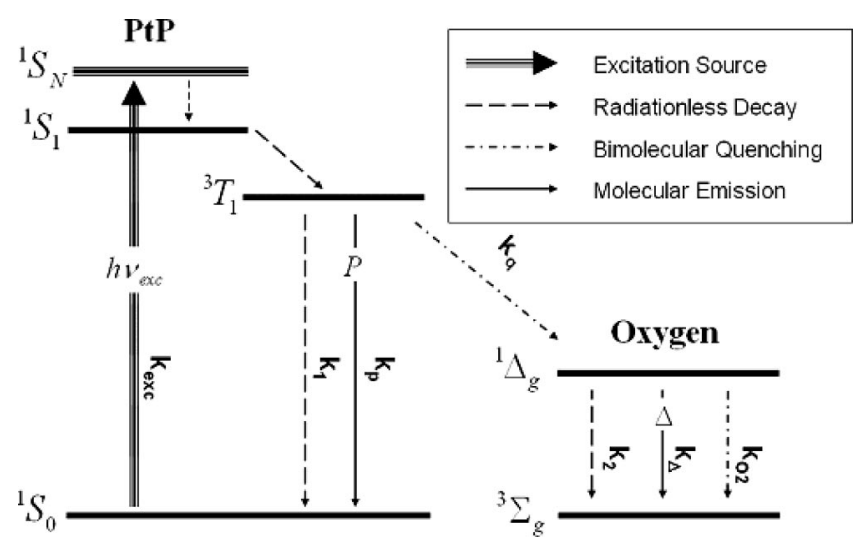

FIG. 1. Energy diagram for PtP and of oxygen. The relevant low energy states for PtP are the ground state ${ }^{1} S_{0}$, excited state ${ }^{3} T_{1}$, and higher excited states ${ }^{1} S_{n}$, and those for oxygen are ground state ${ }^{3} \Sigma_{g}^{-}$and lowest energy excited state ${ }^{1} \Delta_{g}$. Excitation, $h v_{\text {exc }}$, is with $400 \mathrm{~nm}$ laser light. The solid lines with labels $P$ and $\Delta$ are, respectively, the phosphorescence of PtP at $650 \mathrm{~nm}$ and the $1270 \mathrm{~nm}$ oxygen emission; $k_{p}$ and $k_{\Delta}$ are the radiative rate constants corresponding to each emission. The dashed lines are radiationless transitions that do not involve emission; they are labeled with rate constants $k_{1}$ and $k_{2}$. The dot-dashed lines are bimolecular process; $k_{q}$ is ${ }^{3} \Sigma_{g}^{-}$quenching ${ }^{3} T_{1}$ rate constant, and $k_{\mathrm{O}_{2}}$ is ${ }^{1} \Delta_{g}^{-}{ }^{3} \Sigma_{g}^{-}$self-quenching rate constant.

since the excited state ${ }^{1} \Sigma_{g}^{+}$quickly decays into either the excited state ${ }^{1} \Delta_{g}$ or the ground state ${ }^{3} \Sigma_{g}^{-10-12}$ The recent review by Schweitzer and Schmidt provides an excellent discussion of schemes for generation and detection of singlet oxygen. ${ }^{13}$ Several photosensitizer candidates were reported to have $100 \%{ }^{1} \Delta_{g}$ generation efficiency, ${ }^{14,15}$ and work in the field of photodynamic therapy has produced many excellent photosensitizers for singlet oxygen generation. ${ }^{16,17}$ With these and the availability of InGaAs near infrared (NIR) detectors, detection of ${ }^{1} \Delta_{g}$ emission becomes much more feasible even though quantum efficiency is low. ${ }^{18}$ We believe ${ }^{1} \Delta_{g}$ emission can be a probe for direct oxygen concentration measurement and surface pressure mapping, which we shall demonstrate in this article.

Figure 1 outlines the energy transfer diagram between PtP and oxygen. In addition to processes (i)-(v), the further processes related to oxygen emission are as follows:

(vi) $\quad \mathrm{O}_{2}\left[{ }^{1} \Delta_{g}\right] \rightarrow \mathrm{O}_{2}\left[{ }^{3} \Sigma_{g}^{-}\right]+h v[1270 \mathrm{~nm}]:$ Singlet oxygen emission;

(vii) $\mathrm{O}_{2}\left[{ }^{1} \Delta_{g}\right] \rightarrow \mathrm{O}_{2}\left[{ }^{3} \Sigma_{g}^{-}\right]+$heat: Intersystem crossing of oxygen to its ground state; and

(viii) $\mathrm{O}_{2}\left[{ }^{1} \Delta_{g}\right]+\mathrm{O}_{2}\left[{ }^{3} \Sigma_{g}^{-}\right] \rightarrow 2 \mathrm{O}_{2}\left[{ }^{3} \Sigma_{g}^{-}\right]+$heat: Quenching of $\mathrm{O}_{2}\left[{ }^{1} \Delta_{g}\right]$ by $\mathrm{O}_{2}\left[{ }^{3} \Sigma_{g}^{-}\right]$.

Process (vi) is the $1270 \mathrm{~nm}$ emission of interest for this article. Process (vii) is the radiationless intersystem crossing process, and (viii) is the radiationless quenching mechanism of singlet oxygen by ground state oxygen.

In this article we present results for three photosensitizers from the tetra(pentafluoropheny)porphyrin family, namely PtTFPP, MgTFPP, and $\mathrm{H}_{2}$ TFPP. PtTFPP is frequently used in PSP as it has strong phosphorescence yield and no detectable fluorescence. MgTFPP and $\mathrm{H}_{2}$ TFPP, on the other hand, both show moderate fluorescence and very weak phosphorescence. ${ }^{2}$ All three are known to photogenerate singlet oxygen.

\section{EXPERIMENT}

\section{A. Materials}

PtTFPP [platinum tetra(pentafluorophenyl)porphine], MgTFPP [(magnesium tetra(pentafluorophenyl)porphine], and $\mathrm{H}_{2}$ TFPP [(free base tetra(pentafluorophenyl)porphine], were purchased from Frontier Scientific (formerly Porphyrin Products) in Logan, UT. Both PtTFPP and MgTFPP were made by standard porphyrin metallation procedures. Three polymers were used: Polystyrene (PS) beads were purchased from Polysciences, Inc., Warrington, PA. Poly(Bisphenol A-carbonate) polymer (PC) was purchased from Aldrich Chemical Company, Milwaukee, WI. Fluoroacrylic polymer (FIB) was purchased from ISSI, Dayton, OH. Four solvents were used: Dichloromethane CAS 75-09-2 and toluene were purchased from Fisher Scientific Chemicals, Fairlawn, NJ, $\alpha$, $\alpha, \alpha$-trifluorotoluene (TFT) CAS 98-08-8 was purchased from Acros Chemicals, NJ, and chloropentafluorobenzene $\left[\mathrm{C}_{6} \mathrm{~F}_{5} \mathrm{Cl}\right]$, from PCR.

\section{B. Sample preparation}

A solution sample was prepared by dissolving $0.3 \mathrm{mg}$ of PtTFPP in $30 \mathrm{ml}$ of $\mathrm{C}_{6} \mathrm{~F}_{5} \mathrm{Cl}$. The solvent was chosen for its high solubility for oxygen and high yield of singlet oxygen. Thin film samples were made by spin coating a dye-polymer solution onto an aluminum plate. Three stock polymer solutions were prepared in advance of dye-polymer solution. We choose an optimum solvent for each polymer: TFT for FIB, toluene for PS, and dichloromethane for PC. First, 15\% by weight polymer solutions for our respective polymers were prepared by dissolving $3.00 \mathrm{~g}$ of polymer into $20.00 \mathrm{~g}$ of solvent. After a homogeneous polymer solution was obtained, we dissolved an appropriate amount of porphyrin into a small amount of polymer solution to obtain dye-polymer solutions of pre-selected concentration.

The dye-polymer solutions prepared for concentration effect study were 1:50, 1:100, and 1:200 PtTFPP to FIB, where $6.0 \mathrm{mg}$ of PtTFPP was dissolved in $2 \mathrm{~g}$ of the FIB polymer solution for the 1:50, and then by subsequent dilution with the polymer stock solution. Thus 1:100 was made from 1:50, and 1:200 was made from 1:100. For polymer effect study, 1:100 PtTFPP/FIB, 1:200 PtTFPP/PC, and 1:200 PtTFPP/PS were used. The 1:100 PtTFPP/FIB solution was the same sample from concentration study, and the 1:200 PtTFPP/PC and PtTFPP/PS were prepared in similar fashion such that $3.0 \mathrm{mg}$ of PtTFPP was dissolved in $4 \mathrm{mg}$ of the PC or PS polymer stock solution. To make these dye polymer solutions into thin films, we applied $0.5 \mathrm{ml}$ of each solution on to a $1 \times 1$ in. $^{2}$ aluminum plate separately. The solution was allowed to stand for $1 \mathrm{~min}$ to adhere to the aluminum plate, and the plate was spun at $1400 \mathrm{rpm}$ to dry for another minute. The resulting films were of approximately equal thickness after this process, and they were annealed in an oven at $70{ }^{\circ} \mathrm{C}$.

Another thin film sample was prepared by spray painting a 1:200 PtTFPP/FIB 10\% polymer solution on polycarbonate film. This sample was then cut in the shape of " $U W$ " for imaging. 


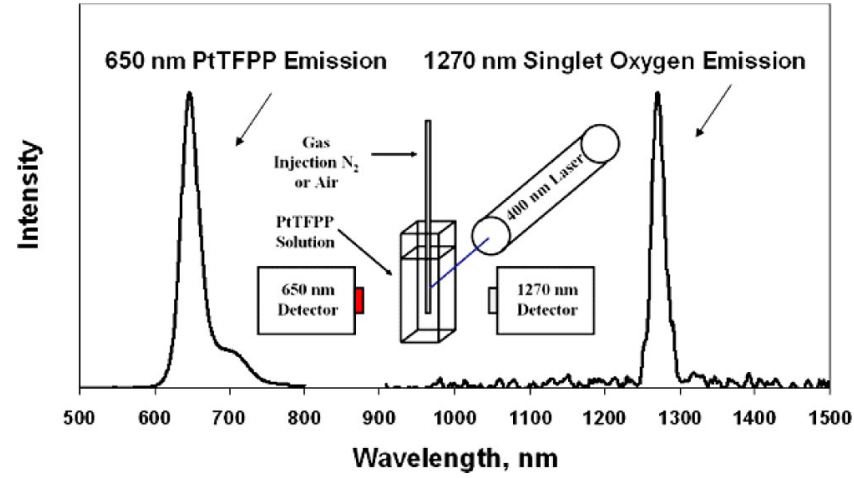

FIG. 2. The normalized emission spectra of PtTFPP and singlet oxygen with experimental setup of solution system in search for cross correlation between $650 \mathrm{~nm}$ emission from PtTFPP phosphorescence and $1270 \mathrm{~nm}$ singlet oxygen emission. The solution system includes PtTFPP dissolved in $\mathrm{C}_{6} \mathrm{~F}_{5} \mathrm{Cl}$ and contained in a cuvette. Dissolved oxygen concentration may be reduced by $\mathrm{N}_{2}$ injection by needle to purge oxygen out. A $400 \mathrm{~nm}$ laser is used to excite PtP, where 650 and $1270 \mathrm{~nm}$ detectors are placed perpendicular to laser path to emission without direct impact of laser.

\section{Instrumentation}

\section{Singlet oxygen lifetime measurement}

Singlet oxygen luminescence lifetimes were measured using the pulses $(532 \mathrm{~nm}, 10 \mathrm{~ns}$, ca. $5 \mathrm{~mJ} /$ pulse $)$ from a Spectra Physics Nd: yttrium-aluminum-garnet laser (GCR-150-30), which excited the polymer film on an Al plate which was inserted into a $10 \times 10 \mathrm{~mm}$ empty quartz cell. The singlet oxygen phosphorescence at $1270 \mathrm{~nm}$ was collected and isolated using a lens and filter system (Interference filter; NB-170-010-B; Spectrogon) and focused into a NIR sensitive photomultiplier tube (PMT) (H9170-45; Hamamatsu). The photocurrent from the PMT was amplified (SR 560, Stanford Research Systems) and stored on a digital oscilloscope (TDS 360, Tektronix). To achieve different oxygen concentrations, the sample cell containing the polymer film was flushed with oxygen/nitrogen mixtures of different ratios for at least $20 \mathrm{~min}$ prior to the measurements. The phosphorescence lifetimes of PtTFPP at $650 \mathrm{~nm}$ were measured using the same instrumentation as described above for singlet oxygen lifetime measurements, except a R928 Hamamatsu photomultiplier tube in conjunction with a monochromator (H10; Jobin-Yvon Inc.) was used as detector.

\section{Solution study}

The experimental setup for the solution correlation study is outlined in Fig. 2. An InGaAs detector, New Focus' Model 2153 IR femtowatt photoreceiver, was used to collect point intensity $1270 \mathrm{~nm}$ emission, and a standard S-20 photomultiplier tube (PMT) was used for the detection of $650 \mathrm{~nm}$ emission intensity; both detectors were fitted with the appropriate bandbass filters. The PtTFPP solution was placed in the cuvette and excited with $400 \mathrm{~nm}$ laser, where both the 1270 and $650 \mathrm{~nm}$ detectors were placed next to the sample. Injecting nitrogen gas to sample to purge ambient oxygen and then allowing $\mathrm{O}_{2}$ to diffuse back into the $\mathrm{N}_{2}$ gas purged solution allowed the oxygen concentration to vary slowly. Data acquisition is achieved using LabVIEW to acquire intensity output under continuous excitation. Figure 2 also displays the emission spectra of the PtTFPP triplet and of the

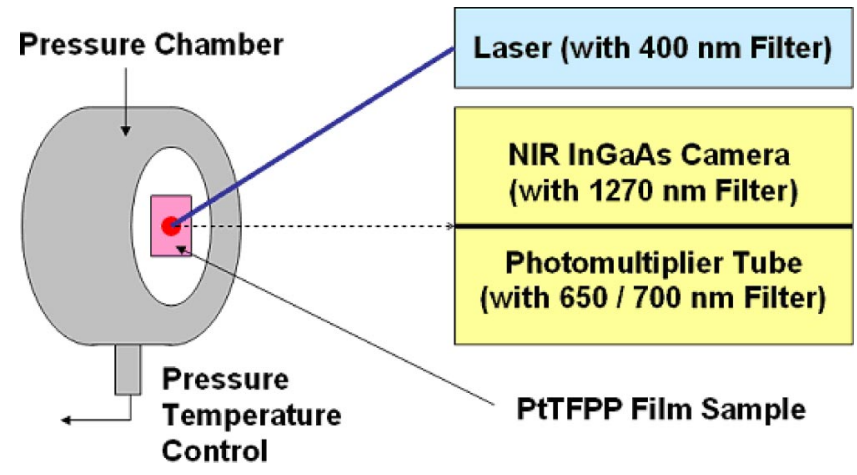

FIG. 3. Experimental setup for Stern-Volmer analysis in pressure chamber: The airfoils of PtTFPP, MgTFPP, and $\mathrm{H}_{2}$ TFPP in different polymers or concentration are placed within a pressure chamber. The chamber has pressure and temperature controls to modulate oxygen concentration and temperature. Porphyrin emissions at 650, $700 \mathrm{~nm}$ and singlet oxygen $1270 \mathrm{~nm}$ emissions are recorded while $400 \mathrm{~nm}$ laser excites the film.

oxygen $\left[{ }^{1} \Delta_{g}\right]$ phosphorescences, which are centered at 650 and $1270 \mathrm{~nm}$, respectively. The singlet oxygen emission spectrum was collected by a NIR spectrometer of Control Development, Model NIR-256L-1.7t and a luminescence Spectrometer LS-50 B from Perkin-Elmer was used to generate the visible emission spectra. Both emissions were stimulated with $400 \mathrm{~nm}$ excitation.

\section{NIR film pressure and temperature measurements}

The apparatus used for evaluating the pressure sensitive paint is shown in Fig. 3, which can measure intensity as function of pressure and temperature. This instrument has a sample chamber that is temperature and pressure controlled. The sample was excited either by a $400 \mathrm{~nm}$ laser Power Technology, LDCU12/5373 or with a $10 \times 10$ array of $395 \mathrm{~nm}$ light emitting diodes (LEDs) (ETG, Inc., Los Angeles, CA, ETG-5UV395-30). The $1270 \mathrm{~nm}$ singlet oxygen emission was imaged with a InGaAs detector, FLIR System's Alpha NIR camera. The camera was fitted with $1280 \mathrm{~nm}$ bandpass filter with $20 \mathrm{~nm}$ full width at half maximum. The instrument allows the operator to choose up to 50 pressure steps. The pressure is set to vacuum and then to atmospheric for initial calibration. A computer controlled valve mechanism sets the desired pressure value. Temperature dependence study was controlled by computer using a thermoelectric module. The module can add or subtract heat and set temperature to the desired value, where temperature is measured by an integrated circuit with $0.1{ }^{\circ} \mathrm{C}$ resolution. Measurement is made from 5 to $45^{\circ} \mathrm{C}$ in $5{ }^{\circ} \mathrm{C}$ increments. Instrumentation for measuring visible signals was discussed in detail previously. ${ }^{19}$ A qualitative pressure experiment was first conducted using the $U W$ PSP film with $400 \mathrm{~nm}$ LED array excitation prior to quantitative pressure and temperature experiments with PSP films.

\section{MODEL}

We here develop a Stern-Volmer-type equation for the use of $1270 \mathrm{~nm}$ singlet oxygen emission to measure oxygen pressure in analogy to the Kavandi equation for using $650 \mathrm{~nm}$ PtP emission to measure oxygen pressure. ${ }^{4}$ The observed phosphorescence intensity, $I$, is directly proportional 

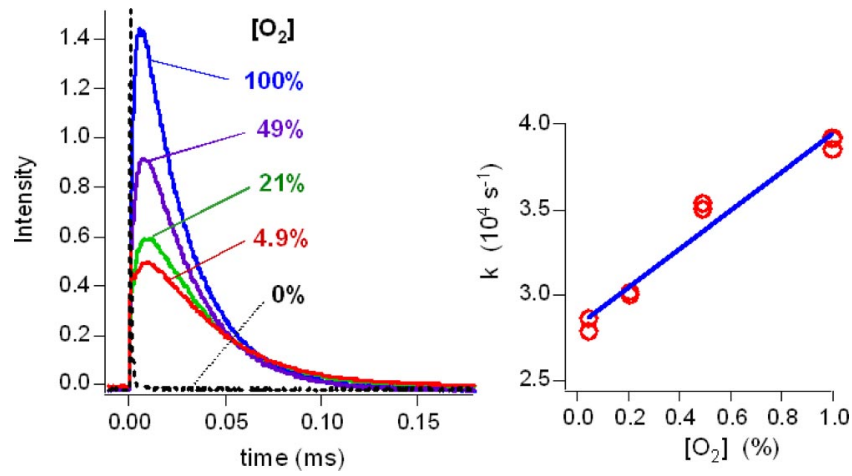

FIG. 4. Left: Kinetic traces of the phosphorescence of singlet oxygen $(1270 \mathrm{~nm})$ generated by excitation of PtTFPP in FIB films with laser pulses at $532 \mathrm{~nm}$ in oxygen-nitrogen mixtures of different ratios at $1 \mathrm{~atm}$. Right: First order rate constant of the decay of the singlet oxygen phosphorescence vs the percentage of oxygen in an oxygen-nitrogen mixture.

to the quantum yield. The ratio $I_{0} / I$ is obtained from the ratio of the intensity at $1 \mathrm{~atm}\left(21 \%\right.$ oxygen) $\left[I_{0}\right]$ to the measured intensity at any other pressure (or oxygen concentration) $[I]$. This intensity ratio corresponds to the quantum yield $\Phi_{0}$ divided by the quantum yield $\Phi$. The quantum yield of an emission is defined as the number of photons emitted divided by the number of photons absorbed. The quantum yield is also given by the rate of radiative decay $\left(k_{P}\right)$ divided by the total rate of decay of the excited triplet PtP molecule.

From Fig. 1 and the definition of quantum yield $\Phi$, we first derive the Kavandi equation for the PtP phosphorescence emission intensity. Here the subscript 0 refers to atmospheric pressure.

$$
\begin{aligned}
\left.\Phi\right|_{650 \mathrm{~nm}}= & \frac{k_{P}}{k_{P}+k_{1}+k_{q}\left[\mathrm{O}_{2}\right]}, \\
\left.\frac{I_{0}}{I}\right|_{650 \mathrm{~nm}}= & \left.\frac{\Phi_{0}}{\Phi}\right|_{650 \mathrm{~nm}}=\frac{k_{P}+k_{1}+k_{q}\left[\mathrm{O}_{2}\right]}{k_{P}+k_{1}+k_{q}\left[\mathrm{O}_{2}\right]_{0}}, \\
\left.\frac{I_{0}}{I}\right|_{650 \mathrm{~nm}}= & \left(\frac{k_{P}+k_{1}}{k_{P}+k_{1}+k_{q}\left[\mathrm{O}_{2}\right]_{0}}\right)+\left(\frac{k_{q}\left[\mathrm{O}_{2}\right]_{0}}{k_{P}+k_{1}+k_{q}\left[\mathrm{O}_{2}\right]_{0}}\right) \\
& \times\left(\frac{\left[\mathrm{O}_{2}\right]}{\left[\mathrm{O}_{2}\right]_{0}}\right),
\end{aligned}
$$$$
\left.\frac{I_{0}}{I}\right|_{650 \mathrm{~nm}}=A+B\left(\frac{\left[\mathrm{O}_{2}\right]}{\left[\mathrm{O}_{2}\right]_{0}}\right),
$$

where $A=\frac{k_{P}+k_{1}}{k_{P}+k_{1}+k_{q}\left[\mathrm{O}_{2}\right]_{0}}$ and $B=\frac{k_{q}\left[\mathrm{O}_{2}\right]_{0}}{k_{P}+k_{1}+k_{q}\left[\mathrm{O}_{2}\right]_{0}}$.

The Stern-Volmer-type equation for singlet oxygen emission from ${ }^{1} \Delta_{g}$ state can be derived in a similar manner from the yield definition, $\left.\Phi\right|_{1270 \mathrm{~nm}}=k_{\Delta} / k_{\Delta}+k_{2}+k_{\mathrm{O}_{2}}\left[\mathrm{O}_{2}\right]$ $\times \Phi_{\Delta} \times \Phi_{T}$, where $\Phi_{\Delta}$ is the ${ }^{1} \Delta_{g}$ state yield of oxygen, and $\Phi_{T}$ is the ${ }^{3} T_{1}$ state yield of PtP. Note that $\Phi_{T}$ is essentially unity for PtP, and $\Phi_{\Delta}$ is $k_{q}\left[\mathrm{O}_{2}\right] / k_{P}+k_{1}+k_{q}\left[\mathrm{O}_{2}\right]$.

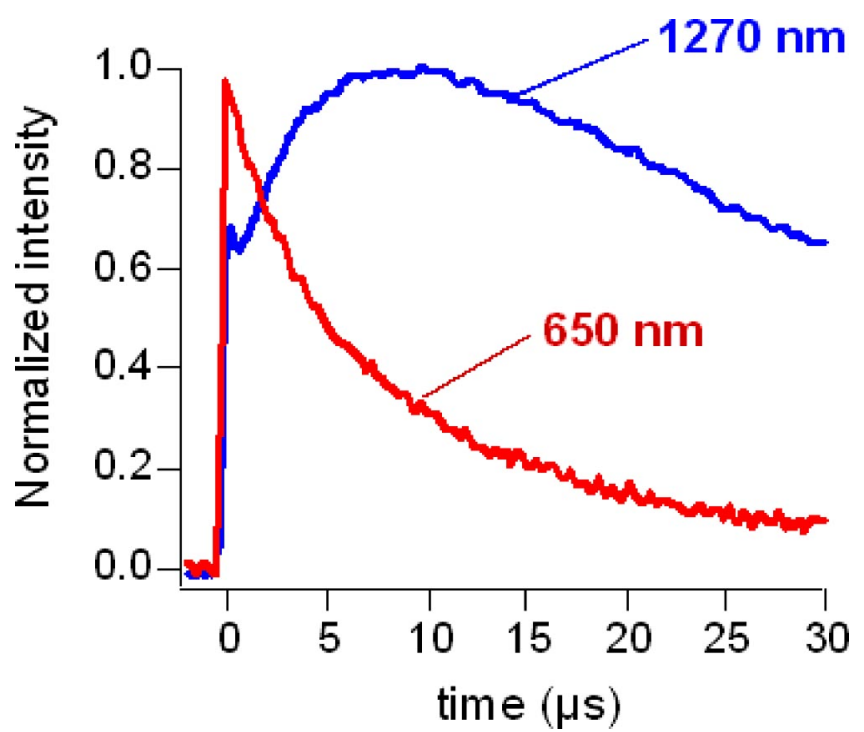

FIG. 5. Kinetic traces of the phosphorescence of PtTFPP (observed at $650 \mathrm{~nm}$ ) and singlet oxygen (observed at $1270 \mathrm{~nm}$ ) generated by excitation of PtTFPP in FIB films with laser pulses at $532 \mathrm{~nm}$ in an air atmosphere (1 atm).

$$
\text { Therefore, } \begin{aligned}
&\left.\Phi\right|_{1270 \mathrm{~nm}}=\left(\frac{k_{\Delta}}{k_{\Delta}+k_{2}+k_{\mathrm{O}_{2}}\left[\mathrm{O}_{2}\right]}\right) \\
& \times\left(\frac{k_{q}\left[\mathrm{O}_{2}\right]}{k_{P}+k_{1}+k_{q}\left[\mathrm{O}_{2}\right]}\right) \text { and } \\
&\left.\frac{I_{0}}{I}\right|_{1270 \mathrm{~nm}=}=\left.\frac{\Phi_{0}}{\Phi}\right|_{1270 \mathrm{~nm}}=\left\{\frac{\left(k_{\Delta}+k_{2}\right)+\left(k_{\mathrm{O}_{2}}\left[\mathrm{O}_{2}\right]\right)}{k_{\Delta}+k_{2}+k_{\mathrm{O}_{2}}\left[\mathrm{O}_{2}\right]_{0}}\right\} \\
& \times\left(\frac{\left[\mathrm{O}_{2}\right]_{0}}{\left[\mathrm{O}_{2}\right]}\right)\left(\frac{k_{P}+k_{1}+k_{q}\left[\mathrm{O}_{2}\right]}{k_{P}+k_{1}+k_{q}\left[\mathrm{O}_{2}\right]_{0}}\right) .
\end{aligned}
$$

Note that the third term of this equation is identical to Kavandi equation. After reducing the first two terms of the equation and substituting for the third, we obtained

$21 \%$ Oxygen

$650 \mathrm{~nm}$
Emission
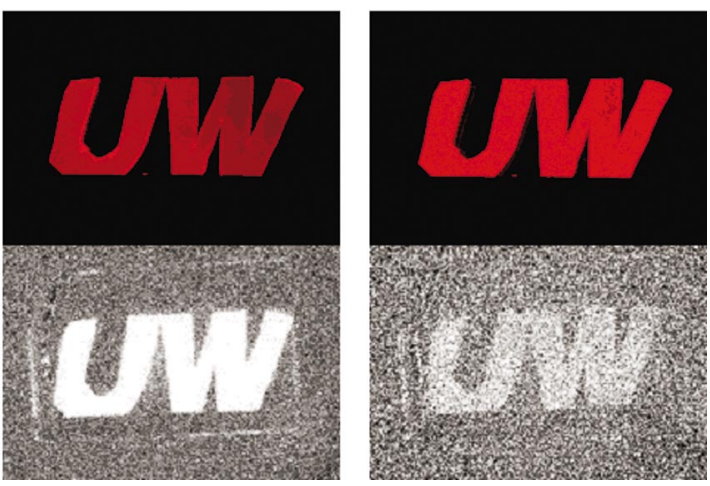

FIG. 6. (Color) Images of 1270 and $650 \mathrm{~nm}$ emissions of UW logo: PSP foil imaged at $21 \%$ oxygen and at $\sim 0 \%$ oxygen, all excited at $400 \mathrm{~nm}$. 


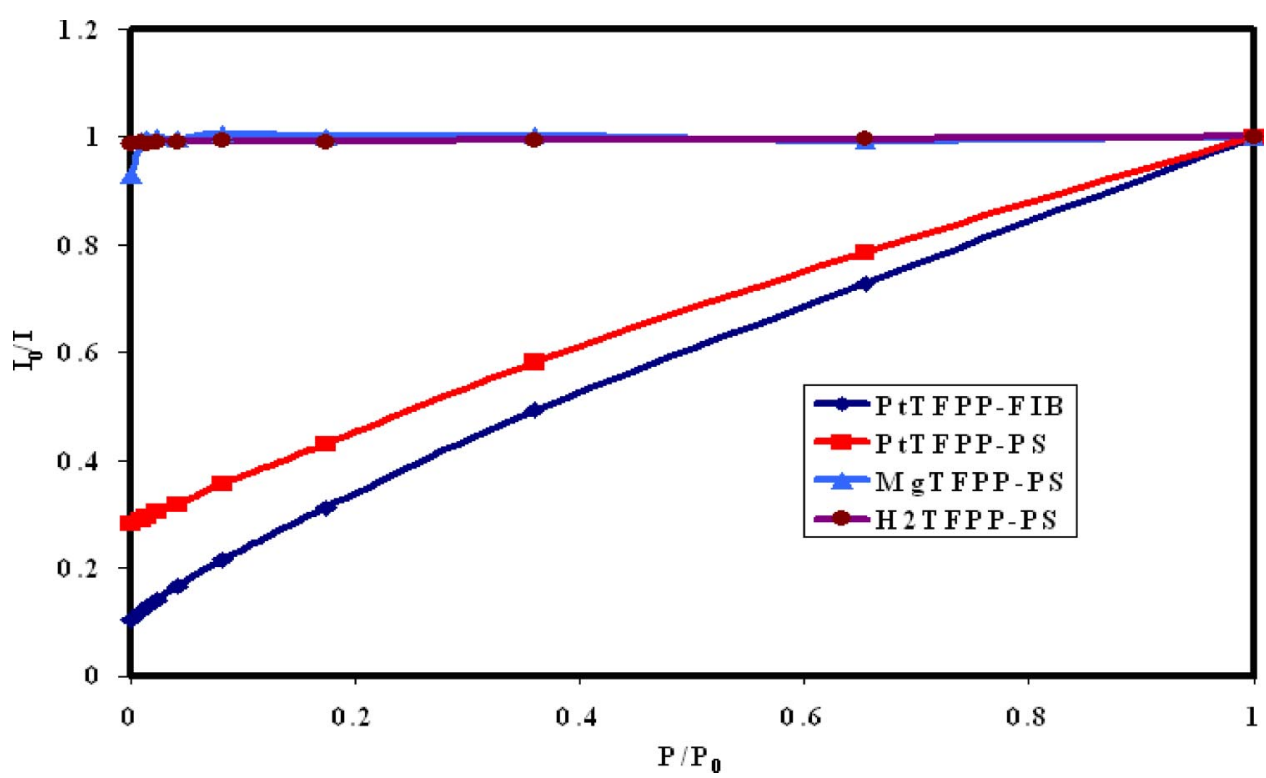

FIG. 7. $I_{0} / I$ vs $P / P_{0}$ Stern-Volmer (Kavandi) plots for PtTFPP in polystyrene (PS) and in FIB polymers measured at $650 \mathrm{~nm}, \mathrm{H}_{2}$ TFPP in PS measured at $700 \mathrm{~nm}$ and MgTFPP in PS measured at $650 \mathrm{~nm}$, all excited at $400 \mathrm{~nm}$.

$$
\begin{aligned}
\left.\frac{I_{0}}{I}\right|_{1270 \mathrm{~nm}}= & \left.\frac{\Phi_{0}}{\Phi}\right|_{1270 \mathrm{~nm}} \\
= & \left\{\left(\frac{k_{\Delta}+k_{2}}{k_{\Delta}+k_{2}+k_{\mathrm{O}_{2}}\left[\mathrm{O}_{2}\right]_{0}}\right)\left(\frac{\left[\mathrm{O}_{2}\right]_{0}}{\left[\mathrm{O}_{2}\right]}\right)\right. \\
& \left.+\left(\frac{k_{\mathrm{O}_{2}}\left[\mathrm{O}_{2}\right]_{0}}{k_{\Delta}+k_{2}+k_{\mathrm{O}_{2}}\left[\mathrm{O}_{2}\right]_{0}}\right)\right\} \\
& \times\left(A+B \frac{\left[\mathrm{O}_{2}\right]}{\left[\mathrm{O}_{2}\right]_{0}}\right)
\end{aligned}
$$

or in short $\left.\frac{I_{0}}{I}\right|_{1270 \mathrm{~nm}}=\left.\frac{\Phi_{0}}{\Phi}\right|_{1270 \mathrm{~nm}}$

$$
=\left(C \frac{\left[\mathrm{O}_{2}\right]_{0}}{\left[\mathrm{O}_{2}\right]}+D\right)\left(A+B \frac{\left[\mathrm{O}_{2}\right]}{\left[\mathrm{O}_{2}\right]_{0}}\right) \text {. }
$$

To modify this relationship to be more Kavandi like, we can take this $1270 \mathrm{~nm}$ ratio and divide it by the Kavandi $650 \mathrm{~nm}$ ratio

$$
\frac{\left.\frac{I_{0}}{I}\right|_{1270 \mathrm{~nm}}}{\left.\frac{I_{0}}{I}\right|_{650 \mathrm{~nm}}}=C \frac{\left[\mathrm{O}_{2}\right]_{0}}{\left[\mathrm{O}_{2}\right]}+D,
$$

where $C=k_{\Delta}+k_{2} / k_{\Delta}+k_{2}+k_{\mathrm{O}_{2}}\left[\mathrm{O}_{2}\right]_{0}$ and $D=k_{\mathrm{O}_{2}}\left[\mathrm{O}_{2}\right]_{0} / k_{\Delta}+k_{2}$ $+k_{\mathrm{O}_{2}}\left[\mathrm{O}_{2}\right]_{0}$. Note that $C+D=1$.

A more useful parameter is the intensity ratio between the oxygen ${ }^{1} \Delta_{g} 1270 \mathrm{~nm}$ emission and the porphyrin ${ }^{3} T_{1}$ $650 \mathrm{~nm}$ emission. Using the yield definitions we can derive the following:

$$
\begin{aligned}
\frac{I_{1270 \mathrm{~nm}}}{I_{650 \mathrm{~nm}}} & =\frac{\left.\Phi\right|_{1270 \mathrm{~nm}}}{\left.\Phi\right|_{650 \mathrm{~nm}}} \\
& =\frac{\left(\frac{k_{\Delta}}{k_{\Delta}+k_{2}+k_{\mathrm{O}_{2}}\left[\mathrm{O}_{2}\right]}\right)\left(\frac{k_{q}\left[\mathrm{O}_{2}\right]}{k_{P}+k_{1}+k_{q}\left[\mathrm{O}_{2}\right]}\right)}{\left(\frac{k_{P}}{k_{P}+k_{1}+k_{q}\left[\mathrm{O}_{2}\right]}\right)},
\end{aligned}
$$

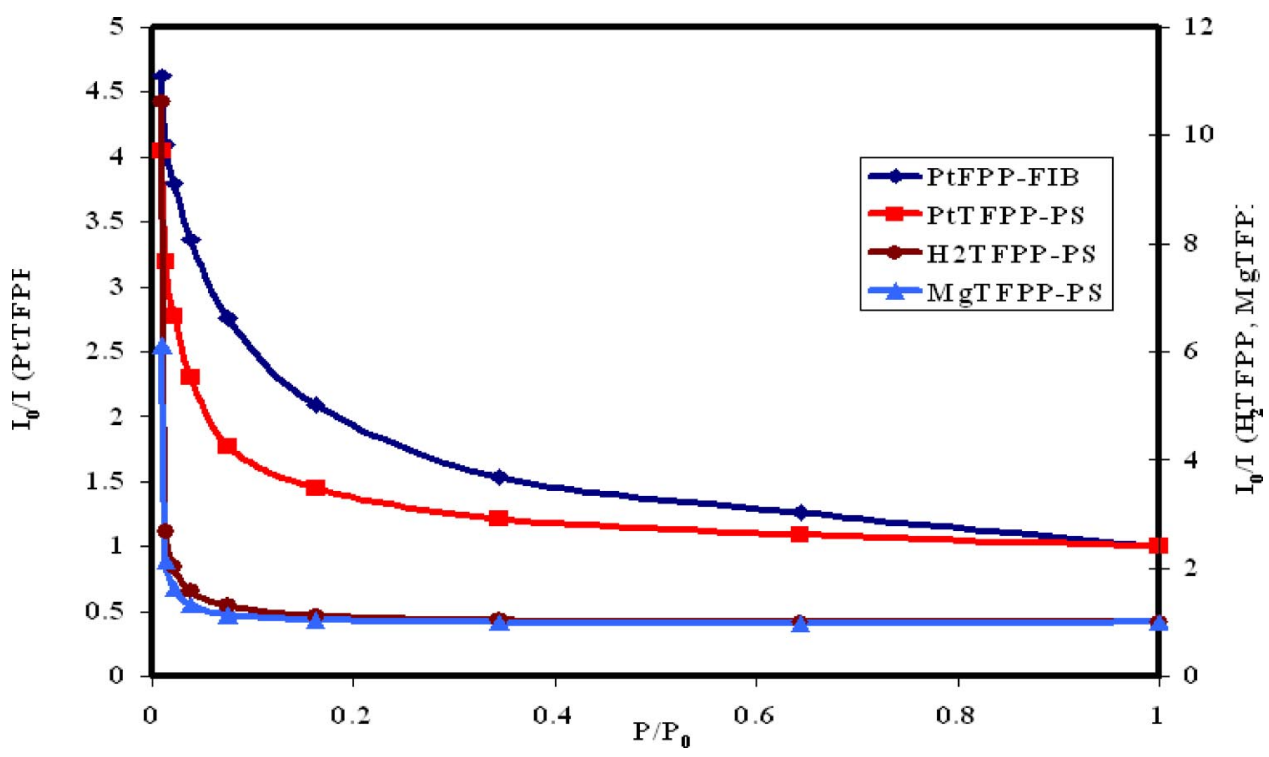

FIG. 8. $I_{0} / I$ vs $P / P_{0}$ Stern-Volmer (Kavandi) plots for PtTFPP in polystyrene (PS) and in FIB polymers, $\mathrm{H}_{2}$ TFPP in PS and MgTFPP in PS, spin-coated at $25^{\circ} \mathrm{C}$, all measured at $1270 \mathrm{~nm}$ all excited at $400 \mathrm{~nm}$. The PtTFPP $I_{0} / I$ data are plotted on the left $Y$ axis, The MgTFPP and $\mathrm{H}_{2}$ TFPP $I_{0} / I$ data are plotted on right $Y$ axis. 


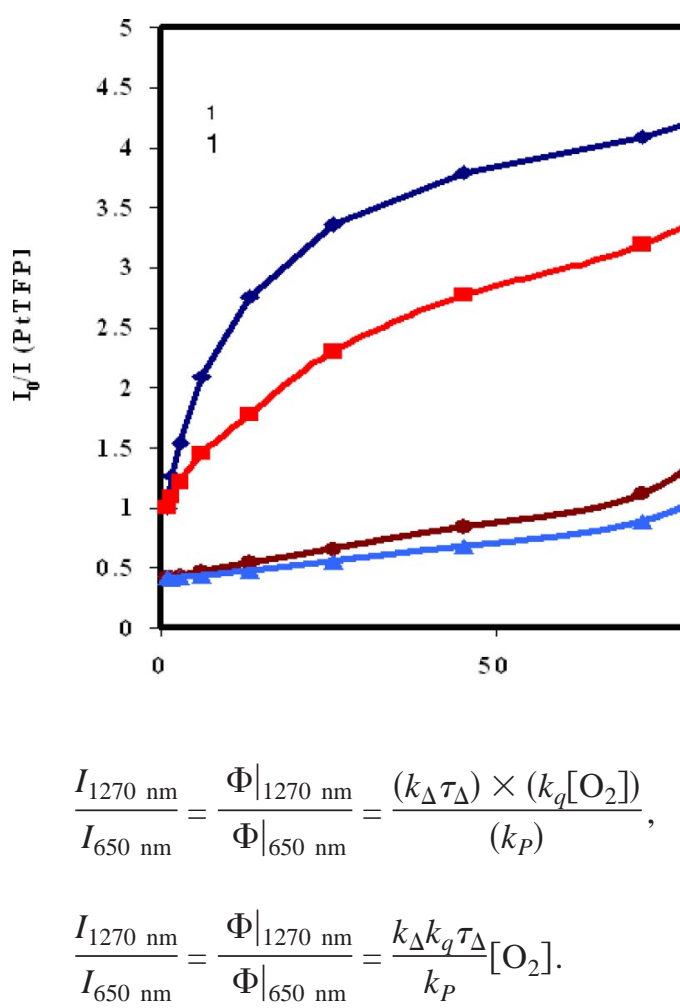

Note that $k_{\Delta}, k_{q}$, and $k_{p}$ are constants for a given platinum porphyrin and polymer combination, and $\tau_{\Delta}$ is the lifetime of singlet oxygen. Krasnovsky and his group observed that $\tau_{\Delta}$ remains constant when varying the oxygen pressure in a measurement in which ${ }^{1} \Delta_{g}$ was produced through a sensitizer in aqueous solution. ${ }^{20}$ We measured the ${ }^{1} \Delta_{g}$ lifetime of PtTFPP in FIB polymer as function of oxygen concentration. Figure 4, left, shows kinetic plots of the singlet oxygen phosphorescence $(1270 \mathrm{~nm})$ at different oxygen concentrations. The observed total rate constant of the decay increases slightly with increasing oxygen concentration as illustrated in Fig. 4, right. The observed singlet oxygen lifetime $\left(\tau_{\Delta}\right.$ $=1 / \mathrm{k}$ ) decreased by $10 \mu \mathrm{s}$ from $0 \%$ to $100 \%$ oxygen and the change from $0 \%$ to $21 \%$ oxygen is only $15 \%$. Therefore, the pressure range of our measurement $\tau_{\Delta}$ can be considered constant. Equation (4) can be simplified to

$$
\frac{I_{1270 \mathrm{~nm}}}{I_{650 \mathrm{~nm}}}=K \times\left[\mathrm{O}_{2}\right],
$$

where $K$ is $k_{\mathrm{O}} \times k_{q} \times \tau_{\Delta} / k_{P}$, with a dimension of [molar $]^{-1}$.

\section{RESULTS AND DISCUSSIONS}

The time evolutions of the phosphorescence of PtTFPP (monitored at $650 \mathrm{~nm}$ ) and the phosphorescence of singlet oxygen (monitored at $1270 \mathrm{~nm}$ ) were studied in more detail using a pulsed laser excitation $(532 \mathrm{~nm}, 10 \mathrm{~ns})$ and a time resolved luminescence detection system. The decay of the PtTFPP phosphorescence at $650 \mathrm{~nm}$ is paralleled by a buildup of singlet oxygen emission at $1270 \mathrm{~nm}$ as illustrated in Fig. 5. The PtTFPP triplet states are quenched by molecular oxygen $\left({ }^{3} \Sigma_{g}^{-}\right)$resulting in singlet oxygen $\left({ }^{1} \Delta_{g}\right)$. Figure 4 (left) shows the decay kinetics of the singlet oxygen phosphorescence at different oxygen concentrations. With increasing oxygen concentrations, the absolute intensity of singlet oxygen phosphorescence increases, because of increased efficiency of PtTFPP triplet quenching by molecular oxygen $\left({ }^{3} \Sigma_{g}^{-}\right)$. The singlet oxygen phosphorescence decay fitted well to pseudo-first order kinetics and the plot of the decay rate

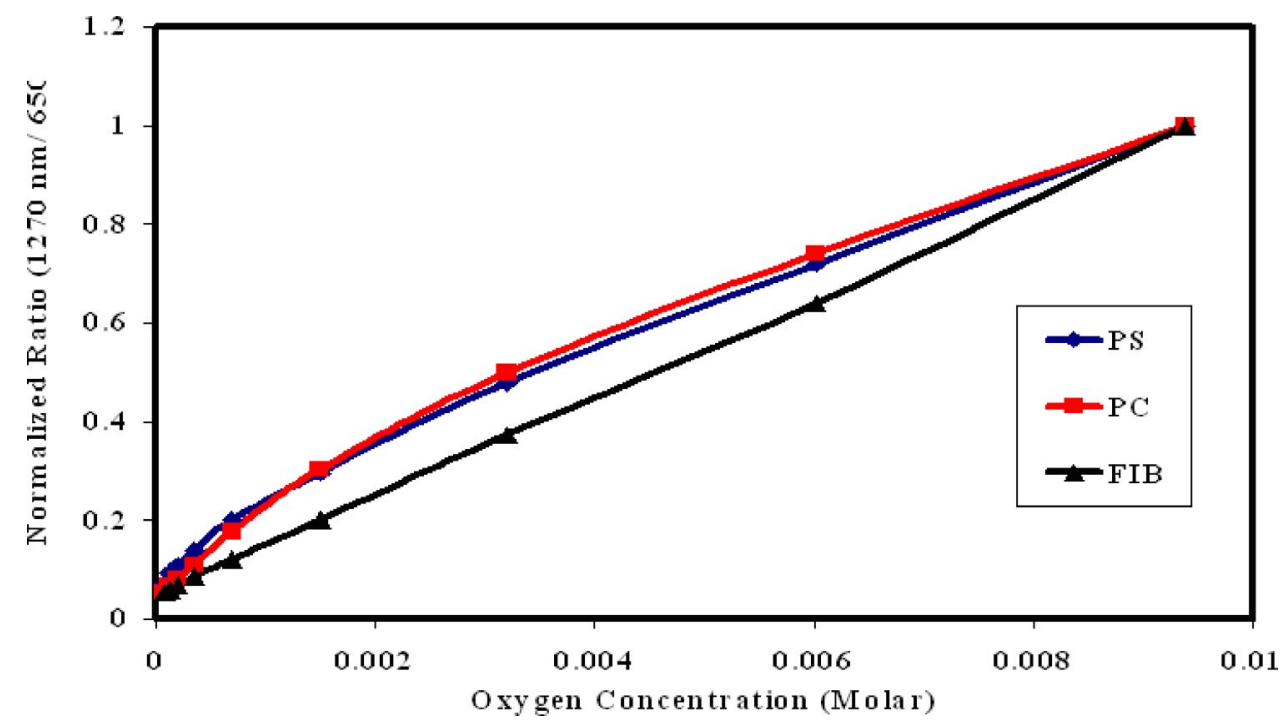

FIG. 10. Normalized intensity ratio plots of the singlet oxygen emission at $1270 \mathrm{~nm}$ to the PtTFPP triplet state phosphorescence at $650 \mathrm{~nm}$ as function of oxygen concentrations in molar. The ratio measured for three films of PtTFPP in FIB, polystyrene (PS) and polycarbonate $(\mathrm{PC})$ all excited at $400 \mathrm{~nm}$. 


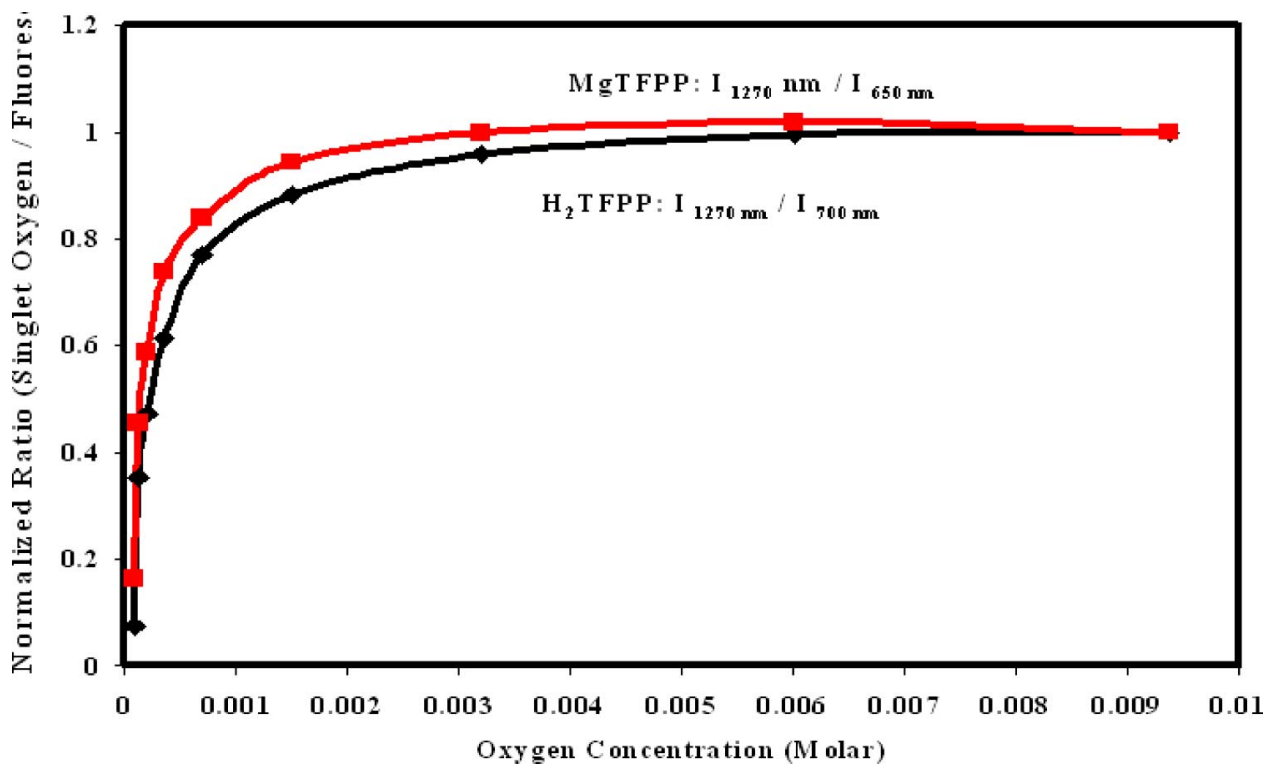

FIG. 11. Normalized intensity ratio plots of the singlet oxygen emission at $1270 \mathrm{~nm}$ to the MgTFPP and $\mathrm{H}_{2}$ TFPP singlet state fluorescence at 650 and $700 \mathrm{~nm}$, respectively as function of oxygen concentrations in molar. The ratio measured for films of MgTFPP and $\mathrm{H}_{2}$ TFPP in polystyrene (PS) all excited at $400 \mathrm{~nm}$. constants versus the oxygen concentrations is shown in Fig. 4 (right). The plot shows that the decay rate constant $\left(1 / \tau_{1 \Delta_{o}}\right)$ increases slightly with increasing oxygen concentrations. The faster decay of the singlet oxygen phosphorescence is attributed to quenching of singlet oxygen $\left({ }^{1} \Delta_{g}\right)$ by molecular oxygen $\left({ }^{3} \Sigma_{g}^{-}\right)$. However, this quenching is negligible at the low oxygen concentrations we used for the following experiments and therefore, $\tau_{1 \Delta_{g}}$ can be considered constant.

Correlation between singlet oxygen and the porphyrin triplet phosphorescence emissions was determined using the experimental setup shown in Fig. 2. The intensities of 650 and $1270 \mathrm{~nm}$ are collected under identical conditions after first purging the solution with $\mathrm{N}_{2}$ gas and then allowing oxygen to diffuse back in over time. The time courses for the two luminescence wavelengths complement each other: The $650 \mathrm{~nm}$ intensity decreases as oxygen concentration increases, while the $1270 \mathrm{~nm}$ intensity increases as oxygen concentration increases. Cross correlation of the two intensities was calculated to be $(-0.997)$. This result motivated us to do further film studies.

Luminescence images of a PtTFPP film in the FIB polymer shaped in the form of "UW" taken at two oxygen concentrations are given in Fig. 6. In Fig. 6, the top shows the $650 \mathrm{~nm}$ emission of PtTFPP phosphorescence emission under air (21\% oxygen) where the emission is low and under near $0 \%$ the luminescence is brighter. In Fig. 6, the bottom shows the $1270 \mathrm{~nm}$ singlet oxygen emission under air $(21 \%$ oxygen) where the emission is bright and under near $0 \%$ oxygen the intensity is low. The four images illustrate the correlations between the quenching of the porphyrin triplet state and the production of oxygen singlet $\left[{ }^{1} \Delta_{g}\right]$ state. The images appear to be exchanged such that the $650 \mathrm{~nm}$ signal has the opposite effect of the $1270 \mathrm{~nm}$ signal as expected. This study shows qualitatively that the concentration of singlet oxygen over the surface of the film can be measured by the oxygen emission intensity as well as by the luminescence quenching of the PtTFPP.

Figure 7 is the standard Stern-Volmer (Kavandi) plot $^{4}$ of
$I_{0} / I$ vs $P / P_{0}[$ Eq. (1)] monitoring the $650 \mathrm{~nm}$ phosphorescence intensity of PtTFPP in films made from two different polymers, polystyrene and FIB. For comparison Fig. 7 also shows the Stern-Volmer plot monitoring the fluorescence intensity of MgTFPP at $650 \mathrm{~nm}$ and $\mathrm{H}_{2}$ TFPP at $700 \mathrm{~nm}$ in polystyrene polymer. All films were measured at ten pressure values and recorded at room temperature. Here the subscript o refers to atmospheric pressure of air ( $21 \%$ oxygen). Ideally the Stern-Volmer curves of PtTFPP are linear as predicted by Stern and Volmer, and in the case of FIB polymer this behavior is observed, ${ }^{5}$ whereas the Stern-Volmer of the MgTFPP and $\mathrm{H}_{2}$ TFPP shows a nearly flat response to pressure. The MgTFPP and $\mathrm{H}_{2}$ TFPP fluorescence has short lifetimes of 9.8 and $5.5 \mathrm{~ns}$, respectively, and is the reason for little or no change of the intensity with pressure. ${ }^{2,15}$

Figure 8 also shows a plot of $I_{0} / I$ vs $P / P_{0}$, but here we monitor the $1270 \mathrm{~nm}$ luminescence of oxygen instead. In this case, $I_{0} / I$ decreases to the right as the intensity of the oxygen $1270 \mathrm{~nm}$ emission increases at higher oxygen pressure. As might be expected, the slopes and curvatures between Figs. 7 and 8 are quite different. It should be noted that the SternVolmer of $1270 \mathrm{~nm}$ for MgTFPP and $\mathrm{H}_{2}$ TFPP shows also a significant intensity change as function of oxygen pressure; their curves are fairly flat for most of the pressure range but changes by tenfold at low oxygen pressure. The results show that oxygen pressure measurements using the $1270 \mathrm{~nm}$ emission can be accomplished using a phosphorescent dye like PtTFPP or a fluorescent dye like MgTFPP and $\mathrm{H}_{2}$ TFPP. The difference in response between these two types of sensitizers can be attributed to the polymer film response time and the corresponding porphyrin triplet state lifetimes. The $90 \%$ response time for the FIB polymer was measured to be $<1 \mathrm{~ms}^{21}$ The phosphorescent lifetimes in the absence of oxygen for PtTFPP, MgTFPP, $\mathrm{H}_{2}$ TFPP are 0.12, 5.6, and $46 \mathrm{~ms}$, respectively. ${ }^{1,2,22,23}$ Thus the long lived triplet state of MgTFPP and $\mathrm{H}_{2}$ TFPP is accessible to oxygen quenching and the production of ${ }^{1} \Delta_{g}$ state is not diffusion limited, which can explain the Stern-Volmer response of the two dyes.

Figure 9 shows a plot of $I_{0} / I$ vs $P_{0} / P$ for the $1270 \mathrm{~nm}$ 
luminescence of oxygen. In this case $I_{0} / I$ increase to the right as the intensity of the oxygen $1270 \mathrm{~nm}$ emission decreases at lower oxygen pressure. Note that PtTFPP curves have wider dynamic range than $\mathrm{H}_{2}$ TFPP and MgTFPP curves. The plots similarly show that $\mathrm{H}_{2}$ TFPP and MgTFPP have greater response at low pressures.

Figure 10 shows a ratio plot of the $1270 \mathrm{~nm}$ singlet oxygen intensity to the $650 \mathrm{~nm}$ triplet porphyrin intensity for PtTFPP in three different polymers as function of oxygen concentration [Eq. (5)]. We note the PtTFPP plot for FIB shows a linear response over the oxygen concentration range and has slope $(K)$ of $100 \mathrm{M}^{-1}$. The ratio of the two correlated signals $I_{1270 \mathrm{~nm}}$ and $I_{650 \mathrm{~nm}}$ produces an enhanced sensitivity measure. Moreover, since the two intensities are being generated from a single luminophor film, the ratio should compensate for illumination variation and angular sensitivity. We anticipate a ratio-metric measurement approach will produce a robust method for determining pressure/oxygen on twodimensional surfaces.

Figure 11 shows a ratio plot of the $1270 \mathrm{~nm}$ singlet oxygen intensity to the 650 and to the $700 \mathrm{~nm}$ fluorescence intensities for MgTFPP and $\mathrm{H}_{2}$ TFPP. The ratio plots show only linear response at the low oxygen concentration range. Similarly, using MgTFPP and $\mathrm{H}_{2}$ TFPP fluorescence signal as intensity reference will produce reliable measurement especially low pressure/oxygen applications.

\section{ACKNOWLEDGMENTS}

The authors would like to thank the Department of Defense Multi-Disciplinary University Research Initiative (MURI) Center on Polymeric Smart Skin Materials through the Air Force Office of Scientific Research Contract No. F49620-01-1-036 under Professor Larry Dalton, and NSF for funding Pressure Sensitive Paint for Insect Flight Research, proposal No. 0352820, under Professor James Callis, both at the University of Washington. The authors at Columbia University thank the NSF Grant CHE 0415516 for support.

${ }^{1}$ D. Eastwood and M. Gouterman, J. Mol. Spectrosc. 35, 359 (1970).

${ }^{2}$ M. Gouterman, The Porphyrins, Optical Spectra and Electronic Structure, edited by D. Dolphin (Academic, New York, 1978), Vol. 3, pp. 1-165.

${ }^{3}$ G. Khalil, M. Gouterman, and E. Green, U.S. Patent No. 4,810,655 (March 7, 1989).

${ }^{4}$ J. Kavandi, J. Callis, M. Gouterman, G. Khalil, D. Wright, E. Green, D. Burns, and B. McLachlan, Rev. Sci. Instrum. 61, 3340 (1990).

${ }^{5}$ M. Gouterman, J. Chem. Educ. 74, 1 (1997).

${ }^{6}$ G. Khalil, M. Gouterman, S. Ching, C. Costin, L. Coyle, S. Gouin, E. Green, M. Sadlek, R. Wan, J. Yearyean, and B. Zelelow, J. Porphyr. Phthalocyanines 6, 135 (2002).

${ }^{7}$ J. H. Bell, E. T. Schairer, L. A. Hand, and R. D. Mehra, Annu. Rev. Fluid Mech. 33, 155 (2001).

${ }^{8}$ L. Tianshu, M. Guille, and J. P. Sullivan, AIAA J. 39, 103 (2001).

${ }^{9}$ O. C. Brown, Ph.D. thesis, Stanford University, 2000.

${ }^{10}$ A. U. Khan, Singlet Molecular Oxygen Spectroscopy: Chemical and Photosensitized, edited by A. A. Frimer (CRC, Boca Raton, FL, 1985), Vol. 1, pp. 39-80.

${ }^{11}$ H. W. Wasserman and R. W. Murray, Singlet Oxygen (Academic, New York, 1979), Vol. 59.

${ }^{12}$ Singlet Molecular Oxygen, edited by A. P. Schaap (Hutchinson and Ross, New York, 1976).

${ }^{13}$ C. Schweitzer and R. Schmidt, Chem. Rev. (Washington, D.C.) 103, 1685 (2003).

${ }^{14}$ A. P. Dramanyan, J. Phys. Chem. A 102, 9833 (1998).

${ }^{15}$ E. Benhur, I. Rosenthal, and C. C. Leznoff, J. Photochem. Photobiol., B 2, 243 (1988).

${ }^{16}$ T. Dougherty, J. Photodynamic Therapy. Photochem. Photobiol. 58, 895 (1993).

${ }^{17}$ L. Santamaria and G. Prino, in Research Progress in Organic Biological and Medicinal Chemistry, edited by U. Gallo and L. Santamaria (Plenum, New York, 1978), Vol. 3.

${ }^{18}$ I. Zebger, J. W. Snyder, L. K. Andersen, L. Poulsen, Z. Gao, J. D. C. Lambert, U. Kristiansen, and P. R. Ogilby, Photochem. Photobiol. 79, 319 (2004).

${ }^{19}$ J. N. Harris, Ph.D. thesis, University of Washington, 1998.

${ }^{20}$ S. Y. Egorov, V. F. Kamalov, N. I. Korotteev, and A. A. Krasnovsky, Chem. Phys. Lett. 163, 421 (1989).

${ }^{21}$ C. M. McGraw, Ph.D. thesis, University of Washington, 2004.

${ }^{22}$ C. M. McGraw, H. Shroff, G. Khalil, and J. Callis, Rev. Sci. Instrum. 74, 5260 (2003).

${ }^{23}$ P. J. Spellane, M. Gouterman, A. Antipas, S. Kim, and Y. C. Liu, Inorg. Chem. 19, 386 (1980). 